\title{
Introducing MARCo: Histoserological Findings of a Multi-Organic Paraneoplastic Syndrome in Cutaneous Melanoma Patients
}

\author{
Mahdy Ranjbar · Armin Mohi · Claudia Pföhler · Salvatore Grisanti • \\ Martin Rudolf
}

Received: July 10, 2016 / Published online: September 1, 2016

(C) The Author(s) 2016. This article is published with open access at Springerlink.com

\begin{abstract}
Introduction: Cutaneous melanoma (CM) is an aggressive skin cancer entity, causing most skin cancer-related deaths. Autoimmune disorders have been described as potential paraneoplastic complications. The purpose of this study was to elucidate the possibility of a combinatory paraneoplastic affection of the retina and cochlea in patients with CM.
\end{abstract}

Methods: Sera samples from CM patients were used for indirect immunofluorescence on histological retinal and cochlear sections.

Enhanced content To view enhanced content for this article go to http://www.medengine.com/Redeem/ 2495F0605C28195A.

M. Ranjbar · A. Mohi · S. Grisanti · M. Rudolf Department of Ophthalmology, University of Lübeck, Lübeck, Germany

M. Ranjbar ( $\square)$

Laboratory for Angiogenesis and Ocular Cell

Transplantation, University of Lübeck, Lübeck,

Germany

e-mail: eye.research101@gmail.com

\section{A. Mohi · M. Rudolf}

Translational Macula Research Laboratory,

University of Lübeck, Lübeck, Germany

C. Pföhler

Department of Dermatology, University Hospital of Saarland, Homburg/Saar, Germany
Furthermore, the serum specimen of a patient with symptomatic affection of both organs was analyzed by multiplex ELISA (enzyme-linked immunosorbent assay) for various cytokines including CD163 (cluster of differentiation 163).

Results: Eleven patients were diagnosed with CM. Autoantibodies against structures of the inner ear were confirmed in all patients who were tested positive for antiretinal antibodies as well. CD163 was significantly elevated in the double-symptomatic patient, who developed metastatic disease.

Conclusion: Paraneoplastic disease of $\mathrm{CM}$ can affect more than one organ and this affect may be correlated with the individual prognosis. Therefore, a thorough anamnesis is needed to avoid missing potential symptoms.

Keywords: Cutaneous melanoma; Inner ear; Paraneoplastic syndrome; Retina

\section{INTRODUCTION}

Cutaneous melanoma $(\mathrm{CM})$ is a potentially aggressive form of skin cancer and its incidence continues to rise with over 250,000 
new cases annually worldwide [1]. CM accounts for the majority of skin cancer-related deaths [1]. A large variance in survival rates, depending on the extent of disease, exists [2]. Fortunately, the previously dismal prognosis of this disease is improving with recent advances in systemic therapy.

$\mathrm{CM}$ derives from neuroectodermal melanocytes. These melanocytes cannot only be found in the skin and hair follicles, but also in the eye and the inner ear. $\mathrm{CM}$ is an immunogenic tumor type, which is frequently associated with spontaneous autoimmune manifestations [3]. In this perspective, the symptoms might be interpreted as an attempt by the immune system to develop antitumor response.

Autoimmune retinopathy has been described as a paraneoplastic manifestation of CM $[4,5]$. Reactive development of antiretinal antibodies with resulting visual impairment is still a poorly understood pathology. Patients describe early symptoms as the presence of sparkles and shimmers (photopsia) as well as blind spots in their vision. On clinical examination, early stages of retinopathy may be difficult to see morphologically, but an electroretinogram (ERG), which measures a standardized evoked response of photoreceptors and subsequent neurons in the retina, is able to demonstrate retinal dysfunction and helps to find the right diagnosis [6].

It has been hypothesized that the inner ear could be a target for a CM-mediated autoimmune response, in which the immune system produces antibodies that cross-react with cells of the organ of Corti, leading to disorders such as vertigo, hearing loss, nausea, and tinnitus [7].

Herein, we aim to demonstrate the histoserological evidence of antibodies against retinal as well as inner ear cells in CM patients. We would like to introduce this condition as MARCo (melanoma-associated retinopathy and cochleopathy).

\section{METHODS}

Patient data as well as blood samples of CM patients in Lübeck (CMPL) and Homburg $(\mathrm{CMPH})$ were evaluated for this study after informed consent. A healthy individual's blood sample was used as non-melanoma control (NMC). The blood was allowed to clot by leaving it undisturbed at room temperature (RT). The clot was removed and following centrifugation the resulting supernatant was collected as designated serum. Aliquots were stored at $-80{ }^{\circ} \mathrm{C}$. To verify the specific binding of antibodies in the sera, we used a commercially available unspecific human IgG (Perbio Science, Bonn, Germany) as antibody control (AC).

Frozen macaque (Macaca fascicularis) retina sections $(8 \mu \mathrm{m})$ were thawed at RT and rehydrated in phosphate buffered saline (PBS). Demineralized paraffin cochlea sections $(10 \mu \mathrm{m})$ from the same animals were deparaffinized and rehydrated in PBS as well. After applying blocking buffer, the slides were incubated with the different probes (AC, NMC, CMPL, CMPH1-10) for $2 \mathrm{~h}$ at RT. The slides were then repeatedly washed and incubated with a biotin-conjugated-goat-anti-human secondary antibody (Rockland, Gilbertsville, PA, USA) for $1 \mathrm{~h}$ at RT. After another washing procedure, slides were incubated with Alexa488-conjugated streptavidin $\quad\left(\lambda_{\mathrm{ex}} / \lambda_{\mathrm{em}}=495 \mathrm{~nm} / 519 \mathrm{~nm} ; \quad\right.$ Life Technologies, Darmstadt, Germany) for $1 \mathrm{~h}$ and 4',6-diamidino-2-phenylindole (DAPI $\lambda_{\mathrm{ex}} /$ $\lambda_{\mathrm{em}}=358 \mathrm{~nm} / 461 \mathrm{~nm}, \quad 1 \mu \mathrm{g} / \mathrm{ml} \quad$ Life Technologies, Darmstadt, Germany) for $10 \mathrm{~min}$ at RT. Finally, the slides were mounted 
using Mowiol mounting medium (Sigma Aldrich, Steinheim, Germany), and images were taken with an inverse fluorescence microscope (Leica DMI6000 B, Leica Microsystems, Wetzlar, Germany) using the same parameters (magnification, exposure

A

Right Eye

Left Eye
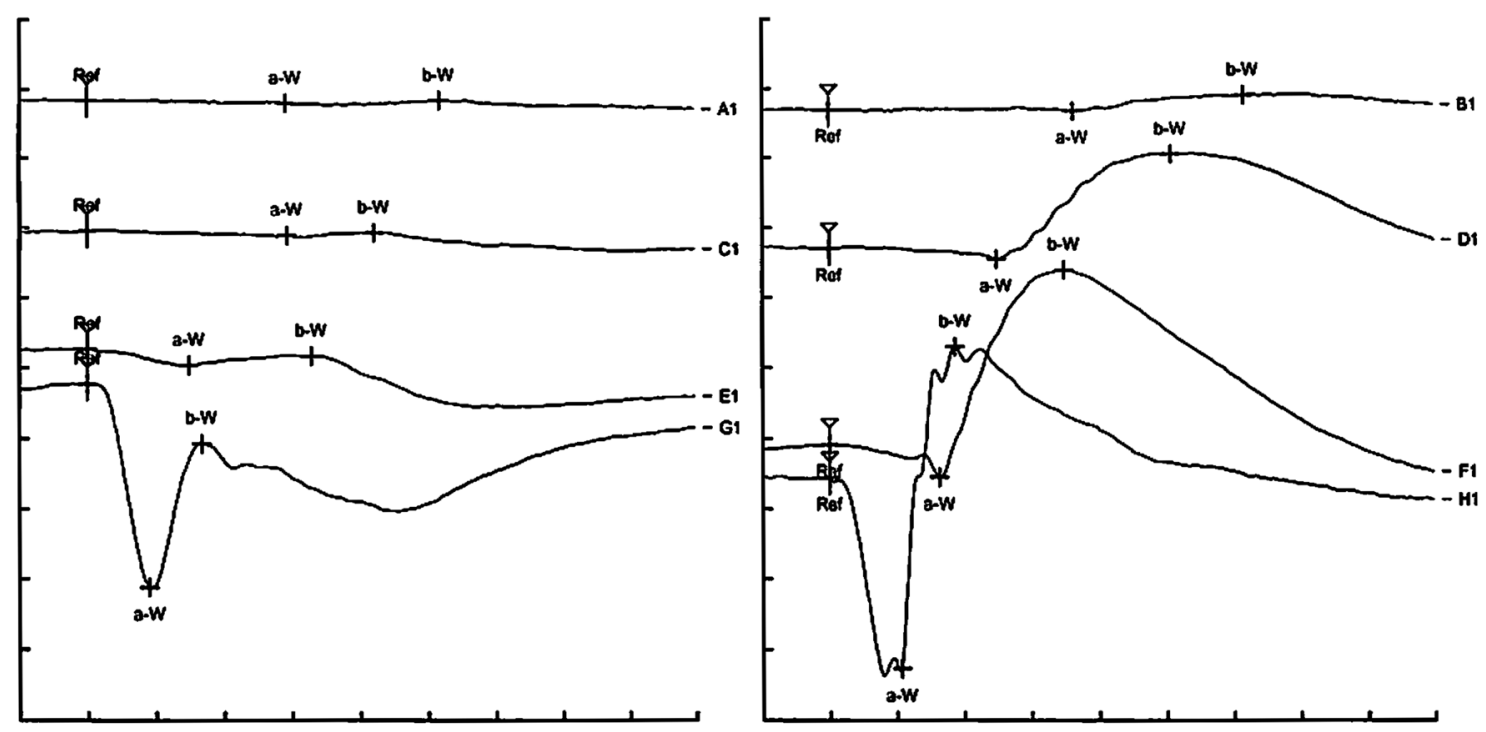

B

Right Eye

Left Eye
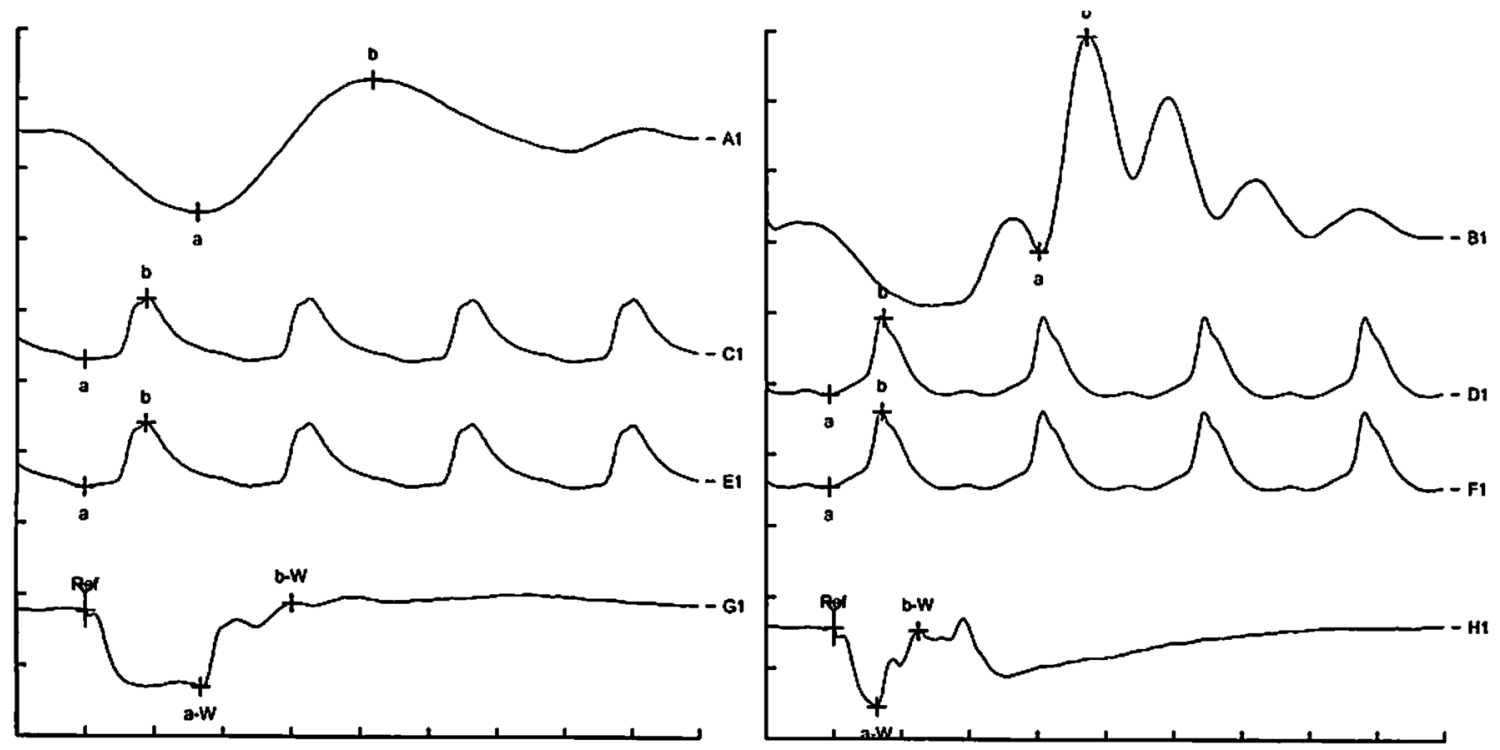

Fig. 1 Characteristic electrophysiological findings in melanoma-associated retinopathy (MAR). a Scotopic (rod responses) full-field electroretinogram (ERG): Negative ERG with missing b-waves on the right eye

and normal responses on the left one. b Photopic (cone responses) full-field ERG: Negative ERG with missing b-waves on both eyes, however, this was more pronounced on the right eye 
time, contrast and saturation) for all specimens. Depending on the tissue (retina vs. cochlea), the same filter and threshold parameters were applied to all images before assessment by semi-quantitative measurement of the number of Alexa488-positive particles, using ImageJ software (Version 1.48b, NIH, Bethesda, USA) in a blinded fashion.

In addition, the NMC and MPL samples were examined for various cytokines (IFN- $\gamma$, IL1-13, IL17, IL23, CD14, CD163, TNFa) using a fully quantitative multiplex ELISA, according to manufacturer's instruction (Q-Plex ${ }^{\mathrm{TM}}$ Human Cytokine Array, Quansys Biosciences, Logan, UT, USA). Data were captured on the Odyssey ${ }^{\circledR}$ infrared imaging system (Li-Cor Biosciences, Lincoln, NE, USA) and analyzed using Quansys Q-view Plus ${ }^{\mathrm{TM}}$ software (Quansys Biosciences, Logan, UT, USA).

All experiments were conducted thrice. Fold-changes relative to NMC are expressed as the mean \pm standard deviation. Statistical analysis was performed with Prism (Version 6, GraphPad, La Jolla, USA) using an unpaired Student $t$ test with $p<0.05$ considered significant. This article does not contain any new studies with human or animal subjects performed by any of the authors.

\section{RESULTS}

All 11 patients were diagnosed with cutaneous melanoma. Seven of them (CMPL, CMPH2, CMPH4, CMPH6-8, CMPH10) had a positive serology (reference laboratory in Homburg, Germany) for melanoma-associated retinopathy (MAR), even though not all of them were symptomatic. When symptomatic, patients were presented with recurrent scintillating scotoma, color vision deficiency, night blindness or visual field constriction.
Fig. 2 Indirect immunofluorescence findings of the retina and cochlea. a Stainings of the retina specimen showed no signal for the isotype antibody control (AC) and almost none for the non-melanoma control (NMC). However, several of our cutaneous melanoma (CM) patients, such as cutaneous-melanoma patient Lübeck (CMPL), showed specific immunofluorescence (Alexa488) around the photoreceptor cell nuclei (ONL) as well as inner/outer segments (IS/OS), the bipolar cells (inner nuclear layer $I N L$ ) and ganglion cells (ganglion cell layer $G C L$ ). In all three panels the retinal pigment cells (RPE) showed typical autofluorescence. Cell nuclei were stained with 4',6-diamidino-2-phenylindole (DAPI, blue). Scale bar is $100 \mu \mathrm{m}$. b Stainings of the cochlea-specimen showed no signal for the isotype $\mathrm{AC}$ and almost none for the non-melanoma control (NMC). However, several of our CM patients, such as CMPL, showed specific immunofluorescence (Alexa488) in the organ of Corti, which consists of inner and outer hair cells (IHC and $\mathrm{OHC}$ ). Furthermore, positive immunofluorescence was captured around Reissner's membrane (RM) and the tectorial membrane (TM). Cell nuclei were stained with DAPI (blue). Scale bar is 100

In most cases, symptoms correlated with electrophysiological findings like for CMPL. In this specific case, the patient was previously diagnosed with two episodes of CM. While fundoscopic examination did not show any pathological changes, the ERG demonstrated a significant b-wave reduction, particularly of the right eye (Fig. 1).

Indirect immunofluorescence on the retinal specimen did show specific staining of the neuronal layers consisting of photoreceptors, bipolar cells, and ganglion cells (Fig. 2a).

As CMPL did describe onset of tinnitus since recurrence of $\mathrm{CM}$, we also performed indirect immunofluorescence on the cochlear specimen. Here, specific stainings of the inner and outer hair cells as well as Reissner's membrane were evident (Fig. 2b). This result was also found to different extents in seven other CM patients. Interestingly, these were the same patients, who had previously been tested positive for MAR (Fig. 3). 

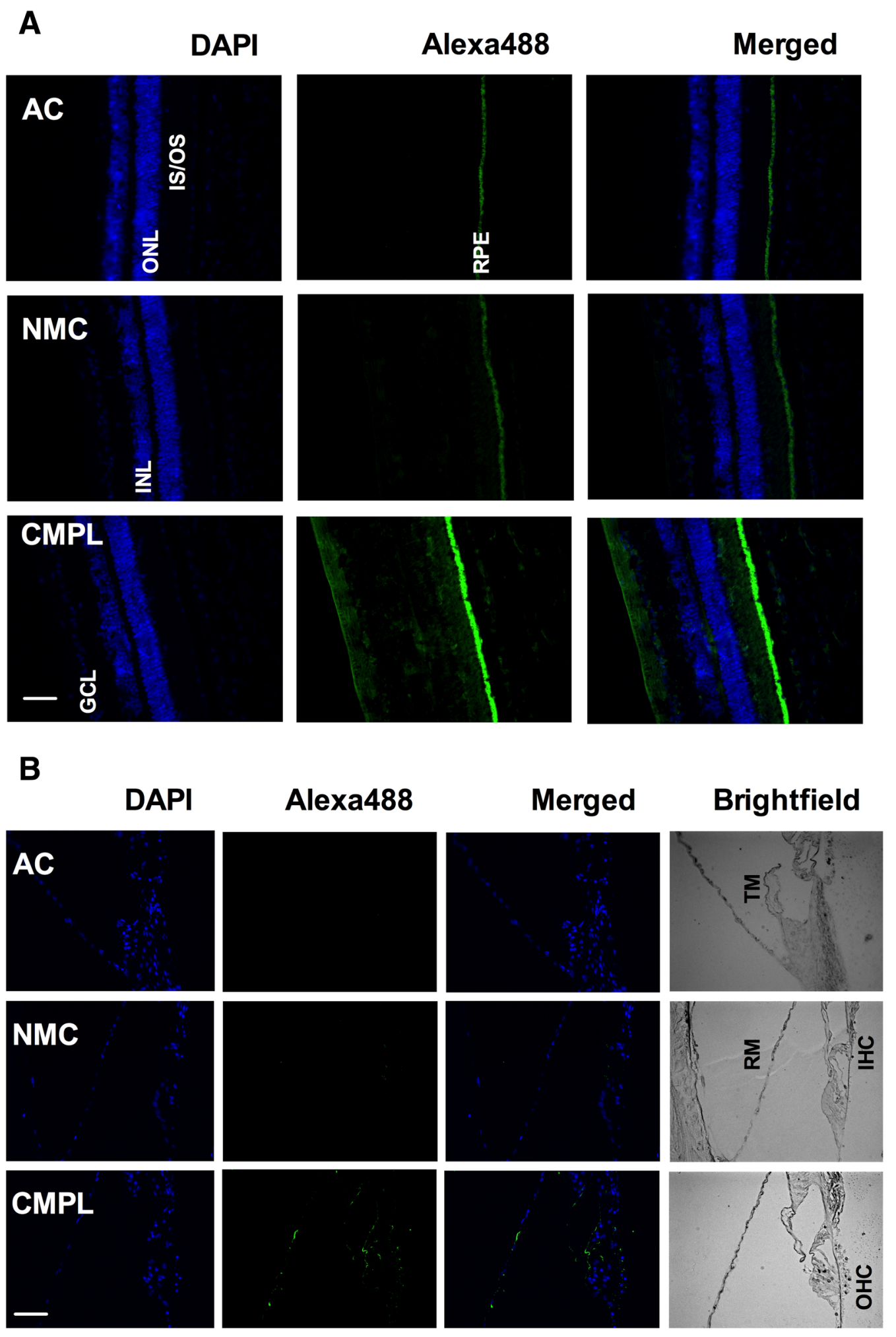


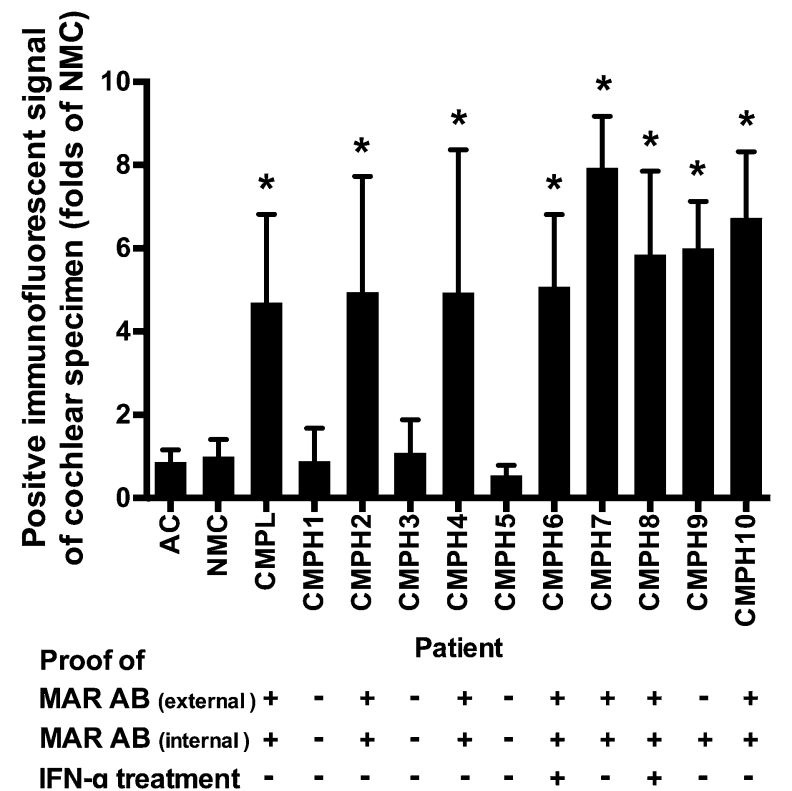

Fig. 3 Results of semi-quantitative evaluation. Values were normalized to NMC. All patients, who were tested positive for antibodies against the retina (MAR AB internal), also showed positive staining on the cochlea slides, in different degrees. Only two patients received IFN- $\alpha$ therapy (CMPH6 and CMPH8). *Signifies $p<0.05$ (unpaired Student $t$ test). Antibody control (AC), non-melanoma control (NMC), cutaneous-melanoma patient Lübeck (CMPL), cutaneous-melanoma patient Homburg $(\mathrm{CMPH})$, melanoma-associated retinopathy antibody proof (MAR AB), interferon- $\alpha$ (IFN- $\alpha$ )

Multiplex ELISA showed a significant elevated serum level of CD163 in CMPL compared to NMC (Fig. 4). All other cytokines were not significantly different.

\section{DISCUSSION}

MAR was first described in 1988 [8]. Most cases have been associated with CM. However, uveal melanoma has also been implicated [5]. Clinical features of MAR typically reflect photoreceptor-mediated dysfunction. Diagnostic confidence is increased by serology, visual function tests, and electrophysiology. The ERG pattern in MAR is classically

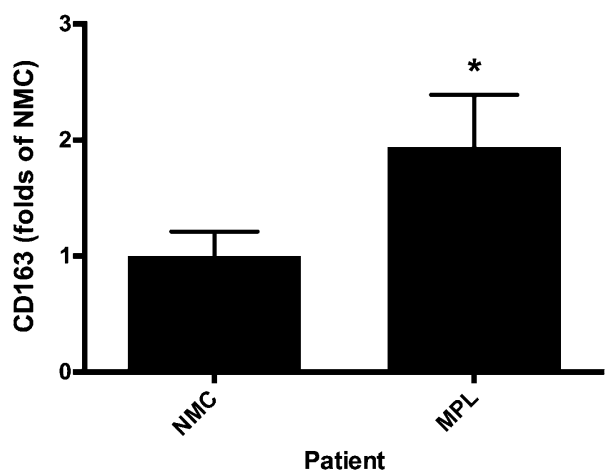

Fig. 4 Cutaneous-melanoma patient Lübeck (CMPL) serum levels of CD163 (Cluster of Differentiation 163). Results were normalized to the non-melanoma control (NMC). This specific cutaneous melanoma patient, who was affected by rapid metastatic disease, showed significant elevated levels of CD163. ${ }^{*}$ Signifies $p<0.05$ (unpaired Student $t$ test)

electronegative. A preserved dark-adapted a-wave, indicating normal photoreceptor function, is followed by a markedly attenuated b-wave, reflecting either bipolar cell dysfunction or disruption of synaptic transmission between photoreceptors and bipolar cells [9].

Anti-bipolar cell antibodies have been considered the characteristic marker of MAR. It has been suggested that these autoantibodies, directed against the postsynaptic receptors of the bipolar cells, interrupt signal transmission with associated photoreceptors [10].

Our results, correlated almost perfectly, except for CMPH9, with those of the external reference laboratory, which suggests a sufficient validity of our method. Negative results of the controls (AC and NMC) underline this fact.

Tinnitus is an abnormal noise perceived in one or both ears, or in the head and is usually associated with damage to the auditory system of the inner ear [11]. Inner ear pathology in the setting of a systemic autoimmune disorder might be immune-mediated. However, isolated immune-mediated inner ear disorders also 
occur. The pathogenesis of immune-mediated inner ear disorders remains largely unknown, although various mechanisms have been suggested such as humoral antibody production, autoreactive $\mathrm{T}$ cells, immune complex deposition, and vasculitis [12] Paraneoplastic audiovestibular disorders associated with CM have already been hypothesized, but not yet confirmed [7].

Despite having confirmed the presence of melanoma-associated autoantibodies against retinal tissue, we have to consider other sources of autoimmunity. Type I interferons, which are mostly $\alpha$-interferons (IFN- $\alpha$ ), are used to treat $\mathrm{CM}$, as it was done in a few of our patients (CMPL, CMPH6, and CMPH8) [13]. Side effects are not uncommon and include exacerbation of pre-existing autoimmune disorders or the de novo induction of autoimmunity. These adverse effects are attributed to the immunomodulatory properties of IFN- $\alpha$, and should be distinguished from autoimmunity associated with melanoma. However, five patients diagnosed with MARCo were not treated with IFN- $\alpha$, which suggests that in this context IFN- $\alpha$ treatment is a weaker confounding factor.

Finally, we aimed at looking whether the presence of multiple autoimmune disorders in melanoma patients might be of prognostic value, because this would allow oncologists to provide appropriate therapy by escalation of the treatment regimen. Various cytokines, such as CD163, have been discussed as potential prognostic indicators [14]. The hemoglobin receptor CD163 is a specific monocyte-macrophage protein, predominantly expressed on anti-inflammatory cell types. It is a promising marker to monitor macrophage activation as macrophages are key cells in the pathogenesis of several infectious, autoimmune, and malignant diseases [15].
Elevated CD163 serum levels have been shown to be a predictor of poor survival in CM, which was also the case in CMPL, who rapidly developed metastatic disease with a fatal outcome [16]. However, further research is needed to confirm the value of melanoma-associated autoimmunity and inflammatory cytokines as a prognostic tool.

\section{CONCLUSION}

To the best of our knowledge this is the first report with histoserological confirmation of melanoma-associated retinopathy and cochleopathy, henceforth known as MARCo. This autoimmune paraneoplastic entity should be addressed during anamnesis as it might be of prognostic significance.

\section{ACKNOWLEDGMENTS}

No funding or sponsorship was received for this study or publication of this article. All named authors meet the International Committee of Medical Journal Editors (ICMJE) criteria for authorship for this manuscript, take responsibility for the integrity of the work as a whole, and have given final approval to the version to be published. The authors would like to thank Christine Örün for excellent technical assistance.

Disclosures. Mahdy Ranjbar, Armin Mohi, Claudia Pföhler, Salvatore Grisanti, and Martin Rudolf have nothing to disclose.

Compliance with Ethics Guidelines. This article does not contain any new studies with human or animal subjects performed by any of the authors. Informed consent was obtained from the patients for using patient data and blood samples. 
Open Access. This article is distributed under the terms of the Creative Commons Attribution-NonCommercial 4.0 International License (http://creativecommons.org/licenses/ by-nc/4.0/), which permits any noncommercial use, distribution, and reproduction in any medium, provided you give appropriate credit to the original author(s) and the source, provide a link to the Creative Commons license, and indicate if changes were made.

\section{REFERENCES}

1. Singh BP, Salama AKS. Updates in therapy for advanced melanoma. Cancers. 2016;8(1):17. doi:10.3390/cancers8010017.

2. Malczewski A, Marshall A, Payne MJ, Mao L, Bafaloukos D, Si L, et al. Intravenous high-dose interferon with or without maintenance treatment in melanoma at high risk of recurrence: meta-analysis of three trials. Cancer Med. 2016;5:17-23.

3. Bouwhuis MG, Ten Hagen TLM, Suciu S, Eggermont AM. Autoimmunity and treatment outcome in melanoma. Curr Opin Oncol. 2011;23:170-6.

4. Pföhler C, Haus A, Palmowski A, Ugurel S, Ruprecht KW, Thirkill CE, et al. Melanoma-associated retinopathy: high frequency of subclinical findings in patients with melanoma. $\mathrm{Br} J$ Dermatol. 2003;149:74-8.

5. Lu Y, Jia L, He S, Hurley MC, Leys MJ, Jayasundera $\mathrm{T}$, et al. Melanoma-associated retinopathy: a paraneoplastic autoimmune complication. Arch Ophthalmol Chic Ill. 2009;1960(127):1572-80.

6. Grange L, Dalal M, Nussenblatt RB, Sen HN. Autoimmune retinopathy. Am J Ophthalmol. 2014;157(266-272):e1.

7. Barozzi S, Ginocchio D, Socci M, Alpini D, Cesarani A. Audiovestibular disorders as autoimmune reaction in patients with melanoma. Med Hypotheses. 2015;85:336-8.

8. Berson EL, Lessell S. Paraneoplastic night blindness with malignant melanoma. Am J Ophthalmol. 1988;15(106):307-11.

9. Keltner JL, Thirkill CE, Yip PT. Clinical and immunologic characteristics of melanoma-associated retinopathy syndrome: eleven new cases and a review of 51 previously published cases. J Neuro-Ophthalmol Off J N Am Neuro-Ophthalmol Soc. 2001;21:173-87.

10. Alexander KR, Fishman GA, Peachey NS, Marchese $\mathrm{AL}$, Tso MO. On response defect in paraneoplastic night blindness with cutaneous malignant melanoma. Investig Ophthalmol Vis Sci. 1992;33:477-83.

11. Sindhusake D, Golding M, Wigney D, Newall P, Jakobsen K, Mitchell P. Factors predicting severity of tinnitus: a population-based assessment. J Am Acad Audiol. 2004;15:269-80.

12. Lunardi C, Bason C, Leandri M, Navone R, Lestani M, Millo E, et al. Autoantibodies to inner ear and endothelial antigens in Cogan's syndrome. Lancet. 2002;21(360):915-21.

13. Sabel MS, Sondak VK. Pros and cons of adjuvant interferon in the treatment of melanoma. Oncologist. 2003;8:451-8.

14. Neagu M, Constantin C, Dumitrascu GR, Lupu AR, Caruntu C, Boda D, et al. Inflammation markers in cutaneous melanoma-edgy biomarkers for prognosis. Discoveries. 2015;31(3):e38.

15. Graversen JH, Svendsen P, Dagnæs-Hansen F, Dal J, Anton G, Etzerodt A, et al. Targeting the hemoglobin scavenger receptor cd163 in macrophages highly increases the anti-inflammatory potency of dexamethasone. Mol Ther. 2012;20:1550-8.

16. Jensen TO, Schmidt H, Møller HJ, Høyer M, Maniecki MB, Sjoegren $\mathrm{P}$, et al. Macrophage Markers in serum and tumor have prognostic impact in American Joint Committee on cancer stage I/II melanoma. J Clin Oncol. 2009;10(27):3330-7. 